\title{
Core-Collapse Supernovae, Neutrinos, and Gravitational Waves
}

\author{
C. D. Ott ${ }^{\mathrm{a}, \mathrm{b}}$, E. P. O’Connor ${ }^{\mathrm{c}}$, S. Gossanª ${ }^{\mathrm{a}}$, E. Abdikamalovª , U. C. T. Gamma ${ }^{\mathrm{a}}$, S. Drasco ${ }^{\mathrm{d}, \mathrm{a}}$ \\ ${ }^{a}$ TAPIR, California Institute of Technology, Pasadena, California, USA \\ ${ }^{b}$ Kavli Institute for the Physics and Mathematics of the Universe, Kashiwa, Chiba, Japan \\ ${ }^{c}$ Canadian Institute for Theoretical Astrophysics, Toronto, Ontario, Canada \\ ${ }^{d}$ Grinnell College, Grinnell, Iowa, USA
}

\begin{abstract}
Core-collapse supernovae are among the most energetic cosmic cataclysms. They are prodigious emitters of neutrinos and quite likely strong galactic sources of gravitational waves. Observation of both neutrinos and gravitational waves from the next galactic or near extragalactic core-collapse supernova will yield a wealth of information on the explosion mechanism, but also on the structure and angular momentum of the progenitor star, and on aspects of fundamental physics such as the equation of state of nuclear matter at high densities and low entropies. In this contribution to the proceedings of the Neutrino 2012 conference, we summarize recent progress made in the theoretical understanding and modeling of core-collapse supernovae. In this, our emphasis is on multi-dimensional processes involved in the explosion mechanism such as neutrino-driven convection and the standing accretion shock instability. As an example of how supernova neutrinos can be used to probe fundamental physics, we discuss how the rise time of the electron antineutrino flux observed in detectors can be used to probe the neutrino mass hierarchy. Finally, we lay out aspects of the neutrino and gravitational-wave signature of core-collapse supernovae and discuss the power of combined analysis of neutrino and gravitational wave data from the next galactic core-collapse supernova.
\end{abstract}

Keywords: core-collapse supernovae, neutrinos, gravitational waves

\section{Introduction}

Core-collapse supernovae (CCSNe), primarily due to their nucleosynthetic impact on the chemical evolution of the universe, are of great importance in astrophysics. Their inner workings or, more precisely, the physical processes that convert the necessary fraction of gravitational energy of collapse into energy of the explosion, involve a broad range of physics, in particular neutrino, nuclear, and gravitational physics. The cores of CCSNe are thus cosmic laboratories for fundamental physics that can be probed by the observation and analysis of neutrinos and gravitational-wave signals from galactic or near-extragalactic CCSNe. This was first and impressively demonstrated by the observation of neutrinos from SN 1987A [1, 2], which confirmed the very basics of CCSN theory [3]: CCSNe are driven by the release of gravitational energy in the collapse of a massive star's core and the vast majority ( $\sim 99 \%)$ of this energy is liberated in $\mathrm{MeV}$ neutrinos.

CCSN theory and modeling have come a long way since the first pioneering numerical studies carried out in the late 1960s and early 1970s [4, 5, 6, 7]. Advances in nuclear and neutrino physics, in combination with improvements in numerical modeling methodology and rapid advances in computer technology have since enabled detailed and self-consistent sphericallysymmetric (1D), axisymmetric (2D), and the first (3D) models of CCSNe (see [8, 9, 10] for reviews).

It is now clear that the prompt hydrodynamic mechanism proposed early on [11] fails robustly. The shock created at core bounce always stalls due to dissociation of infalling heavy nuclei and neutrino losses from the region behind the shock. It must be revived by the explosion mechanism. The (delayed) neutrino mech- 
anism [12] is now believed to drive most CCSN explosions [8]. It relies on the deposition behind the shock (resulting in net energy gain) of a small fraction $(\sim 10 \%)$ of the outgoing $v_{e}$ and $\bar{v}_{e}$ neutrino luminosity within a few hundred milliseconds after core bounce. The neutrino mechanism has been shown to work robustly in spherical symmetry for the lowestmass massive stars (e.g., [13]). Driving explosions in more massive stars with the neutrino mechanism appears to require an interplay of neutrino heating with multi-D fluid dynamics due to neutrino-driven convection and the standing accretion shock instability (SASI) (e.g., [14, 15, 16, 17, 18, 19, 20, 21, 22, 23, 24] and references therein). Convection/SASI leave characteristic imprints on the neutrino and gravitational-wave signals, which, in turn, can be used to observationally probe these instabilities [25, 26, 27, 28, 29, 30].

While garden-variety CCSN explosions (of spectral type II, Ib, Ic) may very well be ultimately explained by the neutrino mechanism, neutrino-driven explosions appear unfit to deliver hyper-energetic explosions (with energies of $\gtrsim 10 \mathrm{~B} ; 1$ [B]ethe $=10^{51} \mathrm{erg}$ ). Such hypernovae are predominantly of the rare Ic-bl subclass (bl stands for relativistically Doppler broadened spectral lines) of CCSNe that has also been associated with longduration gamma-ray bursts (e.g., [31]). Such hyperenergetic CCSN explosions may result from the magnetorotational mechanism. This mechanism relies on rapid progenitor star rotation and efficient amplification of protoneutron star magnetic fields to magnetar strength $\left(\sim 10^{15} \mathrm{G}\right)$ after bounce, launching bipolar jet-like outflows (e.g., [32, 33]). The extreme rotation required in this mechanism may leave characteristic imprints on the neutrino and gravitational wave signal [34, 35].

An interesting potential twist to the standard CCSN story developed with the (re-)discovery of collective neutrino oscillation induced by neutrino-neutrino coherent forward scattering (see, e.g., [36] for a review). Supernova neutrinos, as reviewed by Balantekin in this volume, may oscillate in vacuum, due to the MSW effect [37], and, in regions of high neutrino density, due to collective oscillations. In the inverted neutrino mass hierarchy, collective oscillations are predicted to lead to a nearly complete swap of $v_{e} / \bar{v}_{e}$ spectra with the spectra of heavy lepton $(x=\mu, \tau)$ neutrinos $v_{x} / \bar{v}_{x}$. If this swap occurs below the region in which neutrino heating dominates over neutrino cooling, this would potentially lead to a boost of neutrino energy deposition, since the emission spectra of $v_{x} / \bar{v}_{x}$ are significantly harder than those of their $v_{e} / \bar{v}_{e}$ counterparts and the charged-current absorption cross sections scale with the square of the neutrino energy $\left(\epsilon_{v}^{2}\right)$. That this could indeed have an im- portant impact on neutrino-driven explosions was first shown by Suwa et al. [38]. However, recent detailed oscillation calculations on the basis of realistic supernova background matter distributions and neutrino radiation fields found that collective oscillations are likely to be suppressed in the early post-core-bounce evolution and thus cannot enhance neutrino heating [39, 40, 41] (but see also [42]).

In this contribution to the proceedings of the Neutrino 2012 conferences, we outline, in Section 2, some recent results from multi-D simulations of CCSNe. In Section 3, we discuss how the early postbounce CCSN neutrino signal can be used to constrain the neutrino mass hierarchy. Finally, in Section 4 we give examples of what may be learned from combined observations of neutrinos and gravitational waves from the next nearby CCSN.

\section{Results from Recent Neutrino-Driven Multi- Dimensional Core-Collapse Supernova Models}

The first set of detailed 2D CCSN simulations carried out in the mid 1990s (e.g., [43, 44, 45]) demonstrated that the negative entropy gradient created by neutrino heating leads to vigorous convection in the gain region behind the shock (where neutrino heating dominates over cooling). The added degree of freedom in 2D liberates the infalling gas to move not just in the radial direction (as in 1D), but also laterally. As a consequence, the dwell time in the gain region of a given accreting mass element is increased, leading to greater total neutrino heating and high-entropy turbulent flow that pushes the shock to larger radii and aids the explosion.

The standing accretion shock instability (SASI; e.g., [46, 47, 48]) is another hydrodynamic instability of relevance in the postbounce evolution of CCSNe. In 2D simulations, it drives large-scale periodic low-order ( $\ell=1$ is the fastest growing mode) deformations of the shock front, which, in the nonlinear phase of the instability, lead to larger average shock radii, secondary shocks, and also increase the dwell time of material in the gain region (e.g., [47, 14, 16, 17]). In 3D, nonaxisymmetric $(|m|>0)$ modes will develop. If rotation is present, $m \pm 1$ spiral modes may appear (e.g., [49]), while in the nonrotating case all $m$ of a given $\ell$ are degenerate and should grow to comparable amplitudes, effectively spreading the typical $2 \mathrm{D} \ell$ amplitude over its $m$-modes in 3D [50, 22, 21, 23].

There has been much recent discussion in the literature about the respective roles of neutrino-driven convection and SASI and about which of the two instabilities is the primary instability (i.e., the one that grows 


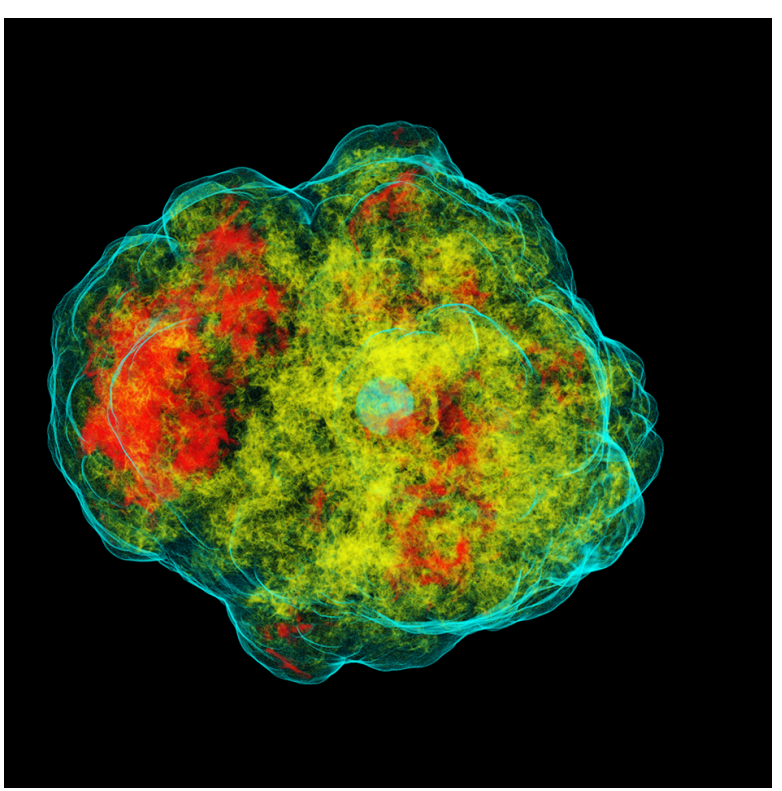

Figure 1: 3D volume rendering of the specific entropy $s$ at $\sim 150 \mathrm{~ms}$ after core bounce in a 3D general-relativistic simulation carried out by Ott et al. [23]. The scale of the frame is $700 \mathrm{~km}$ on a side. The colormap is chosen such that cyan correesponds to a moderate $s \sim 4 \mathrm{k}_{\mathrm{B}}$ baryon $^{-1}$. Yellow and red indicate regions with $s \sim$ $16 \mathrm{k}_{\mathrm{B}}$ baryon $^{-1}$ and $s \sim 20 \mathrm{k}_{\mathrm{B}}$ baryon ${ }^{-1}$, respectively.

first and is dominant) [16, 17, 19, 22, 21, 20, 23]. While this is not the place to review this discussion in detail, it is worth pointing out a few key aspects:

(i) Which instability grows first depends on (a) the early postbounce rate of advection through the region of convective instability (and, hence, on the accretion rate set by progenitor star structure) and $(b)$ on the size of the seed perturbations present in the flow from which convection may grow [48, 47] and thus on the level of asphericity in the last stages of nuclear burning (e.g., [51]). If the initial size of a perturbation is too small to develop into a bouyant plume before it is advected out of the region of convective instability, then convection cannot grow and SASI will dominate (at least initially) [47, 17].

(ii) When the SASI approaches its nonlinear phase, it leads to secondary shocks and seeds convection (e.g., [47]), which, in turn, may be limiting further SASI growth (e.g., [52]).

(iii) The behavior of the SASI appears fundamentally different in 3D and 2D. In 2D, the dominant $\ell=$ 1 mode leads to strong periodic north-south sloshing motions of the shock that persist even when neutrinodriven convection becomes strong. In $3 \mathrm{D}, \ell=1$ is still the fastest growing mode. However, its power is equipartitioned into its $m=\{0, \pm 1\}$ modes, the invidi- ual $(\ell, m)$ amplitudes stay at much smaller values than in $2 \mathrm{D}$ and no large-scale single sloshing mode develops [50, 18, 29, 22, 21, 23].

(iv) In 3D, if neutrino-driven convection gets a chance to grow, it appears to robustly become the dominant instability and quench the SASI, in particular in simulations that have strong neutrino heating and show explosions [22, 21, 23]. In the latter, non-oscillatory, high-amplitude $\ell=1, m=\{0, \pm 1\}$ deformations appear that correspond to large bubbles of high-entropy that push out the shock front in a particular direction [22, 21, 23, 53]. An example of this is shown in Fig. 1. which depicts a volume rendering of the specific entropy at $\sim 150 \mathrm{~ms}$ after core bounce in a simulation of Ott et al. [35].

Nature is 3D. While highly detailed 2D multi-group radiation-hydrodynamics simulations are now available [16, 17], a full understanding of CCSN hydrodynamics must await equally detailed 3D simulations, which have not been performed, but rapid progress towards them is being made (e.g., [54, 55, 23]).

\section{Supernova Neutrinos as Probes of the Neutrino Mass Hierarchy}

Recently, Serpico et al. [56] proposed that the neutrino mass hierarchy could be determined from the early postbounce neutrino signal of the next galactic CCSN for which complicated collective oscillation effects may be ignored [39, 40]. The original flavor (at emission) of $\bar{v}_{e}$ arriving at a detector on Earth depends on the neutrino mass hierarchy. For the normal hierarchy $(\mathrm{NH})$, the MSW effect [37] in the outer regions of the star leads to an incomplete conversion of $\bar{v}_{e}$ to heavy-lepton antineutrinos $\left(\bar{v}_{x}\right)$ and vice versa. For this hierarchy, in the adiabatic approximation for neutrino oscillations, the $\bar{v}_{e}$ signal at Earth is a linear combination of the original $\bar{v}_{e}$ signal $\left(\propto \cos \left(\theta_{12}\right)^{2} \sim 0.71\right)$ and the original $\bar{v}_{x}$ signal $\left(\propto \sin \left(\theta_{12}\right)^{2} \sim 0.29\right)$. However, for the inverted hierarchy $(\mathrm{IH})$ the $\bar{v}_{e}$ signal at Earth is completely composed of the original $\bar{v}_{x}$ signal [57]. So, for the two hierarchies, we have:

$$
\begin{aligned}
& L_{\bar{v}_{e}, \oplus}^{\mathrm{NH}}=\cos \left(\theta_{12}\right)^{2} L_{\bar{v}_{e}, \mathrm{SN}}+\sin \left(\theta_{12}\right)^{2} L_{\bar{v}_{x}, \mathrm{SN}}, \\
& L_{\bar{v}_{e}, \oplus}^{\mathrm{IH}}=L_{\bar{v}_{x}, \mathrm{SN}} .
\end{aligned}
$$

The distinguishing characteristic explored in [56], and further in this proceedings contribution, is the difference in the rise times of the $\bar{v}_{e}$ signal and the $\bar{v}_{x}$ signal. The production of $\bar{v}_{e}$ is initially suppressed due to the large electron chemical potential before bounce 


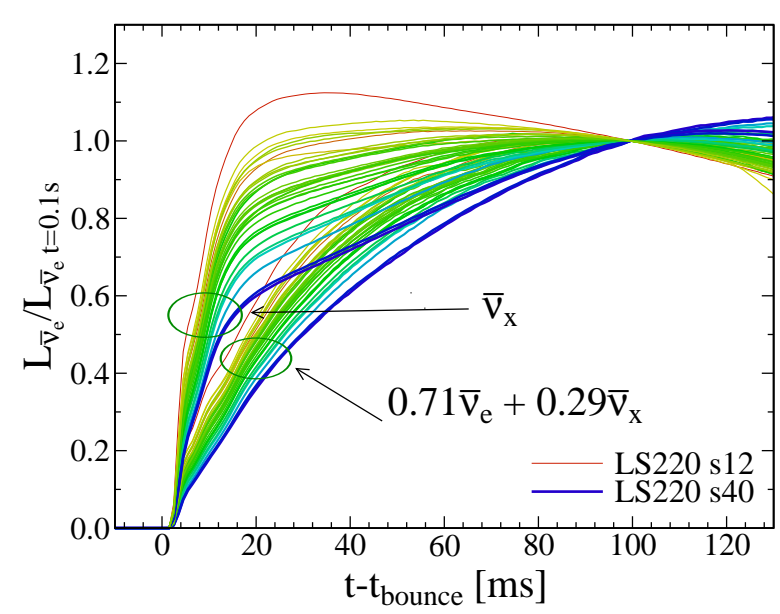

Figure 2: Normalized (to the luminosity at $100 \mathrm{~ms}$ after bounce) electron antineutrino $\left(\bar{v}_{e}\right)$ luminosities at Earth for 32 progenitor star models and assuming both the inverted (family of curves labeled via $\bar{v}_{x}$ ) and normal (family of curves labeled $0.71 \bar{v}_{e}+0.29 \bar{n} \bar{u}_{x}$ ) hierarchy. The colors distinguish progenitor models, colors ranging from red $\rightarrow$ yellow $\rightarrow$ green $\rightarrow$ blue denote increasing compactness, Eq. (3] [58].

and in the very early postbounce. It is not until the degeneracy has been partially lifted in the outer regions of the protoneutron star that significant $\bar{v}_{e}$ luminosity emerges from the supernova. Hence, $\bar{v}_{e}$ have a slow rise time. Heavy-lepton neutrino production does not undergo this suppression and the $\bar{v}_{x}$ luminosity rises much more quickly. In the following, we explore whether a high-event-rate detector like IceCube (e.g., [59]) will be able to distinguish between the two expected neutrino signals given by eqs. (1) and (2). In particular, we extend the analysis of [56] and explore whether there exist degeneracies in the observed signal with progenitor star model and equation of state (EOS). We use results of O'Connor \& Ott [58 $]^{1}$ who simulate the preexplosion phase in 32 solar-metallicity progenitor stars with masses ranging from 12 to $120 M_{\odot}$ from Ref. [60]. Two equations of state are used, the LS220 EOS from [61] and the HShen EOS from [62]. In Fig. 2, we show the normalized (to its value at $100 \mathrm{~ms}$ after bounce ) $\bar{v}_{e}$ luminosities expected at Earth assuming both the normal (slowly rising curves) and inverted hierarchies (fast rising curves). We show only the luminosities for the LS220 EOS. The fast rise time of the inverted hierarchy is clearly visible in the data. This figure is very similar to Fig. 4 of [56], but shows a larger range of progenitors, which we parameterize by their compactness parameter,

$$
\xi_{M}=\left.\frac{M / M_{\odot}}{R\left(M_{\text {bary }}=M\right) / 1000 \mathrm{~km}}\right|_{t=t_{\text {bounce }}},
$$

\footnotetext{
${ }^{1}$ Available at http://www.stellarcollapse.org/M1prog
}

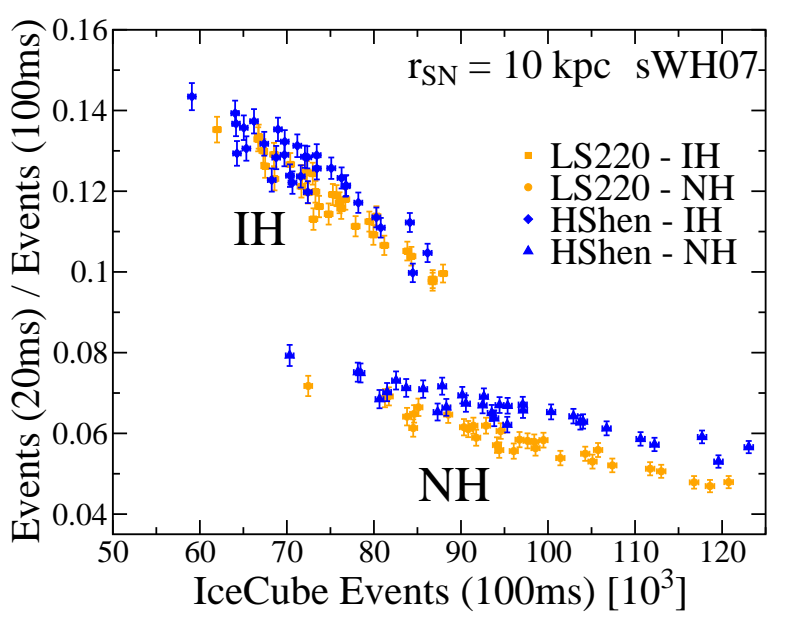

Figure 3: Ratio of the number of expected events detected in the first $20 \mathrm{~ms}$ to the number of expected events detected in the first $100 \mathrm{~ms}$ versus cumulative events at $100 \mathrm{~ms}$. We show this ratio for each progenitor star model, EOS, and most important for our discussion, the assumed neutrino mass hierarchy. The fast rise time of the inverted hierarchy signal gives a large ratio for all models, independent of EOS and progenitor star details. Note that neither axis begins at zero. Error bars are purely statistical and include the contribution from the IceCube background rate of $\sim 2200$ events per $1.6384 \mathrm{~ms}$ bin.

where $R\left(M_{\text {bary }}=M\right)$ is the radial coordinate that encloses a baryonic mass of $M$ at the time of core bounce. Here, we use $M=1.75 M_{\odot}$, since this is a fiducial mass scale in the postbounce preexplosion phase [58]. For clarity, the color scale in Fig. 2, ranging from red $\rightarrow$ yellow $\rightarrow$ green $\rightarrow$ blue, denotes increasing progenitor star compactness [58].

We predict interaction rates in IceCube by using the following formula [35], $R_{\bar{v}_{e}}^{\mathrm{IC}} \sim 2.6 \times 10^{4} \mathrm{~s}^{-1} L_{\bar{v}_{e}, 51}^{\oplus} / r_{10 \mathrm{kpc}}^{2}$, where $L_{\bar{v}_{e}, 51}^{\oplus}$ is the $\bar{v}_{e}$ luminosity at Earth in units of $10^{51} \mathrm{erg} \mathrm{s}^{-1}$ and $r_{10 \mathrm{kpc}}$ is the distances in units of $10 \mathrm{kpc}$, the fiducial galactic distance. We quantitatively discriminate between the two signals at Earth by taking the fluence ratio of the number of events in the first $20 \mathrm{~ms}$ to the number of events in the first $100 \mathrm{~ms}$. While we have not performed an analysis to find the optimal times to evaluate the fluence ratio, $20 \mathrm{~ms} / 100 \mathrm{~ms}$ is a reasonable choice, as one can see in Fig. 2 It is apparent that by $20 \mathrm{~ms}$ the $\bar{v}_{x}$ luminosity has begun to level off. If we picked a time smaller than $20 \mathrm{~ms}$, we would start to be dominated by statistical error for the number of observed events. Times in the numerator longer than $20 \mathrm{~ms}$ lead to fluence ratios that are increasingly converging towards one. The inverted hierarchy, which gives a faster rising signal in $\bar{v}_{e}$ at Earth, will have a larger fluence ratio than the normal hierarchy. We show this ratio in Fig. 3 for each progenitor model, EOS, and 
hierarchy. For clarity, we choose to plot the fluence ratio as a function of total number of event $2^{2}$ detected at $100 \mathrm{~ms}$. The results of our extended model set confirm the results of obtained by [56]. The normal and inverted hierarchies should be distinguishable from the rise time of the early neutrino signal from the next galactic CCSN in an IceCube-like detector. In addition, over the range of progenitors that we explore and the two quite distinct EOS we employ, the signals predicted by the two hierarchies remain clearly distinct, strengthening the ability to use supernovae neutrino rise times as a probe of the neutrino mass hierarchy.

\section{The Next Galactic Core-Collapse Supernova: Gravitational Waves and Neutrinos Probing the Supernova Engine}

The next galactic CCSN is guaranteed to come. While the predicted rates for galactic CCSNe are in the range of a disheartening one per $\sim 30-100$ years [63, 64], we prefer to look at this from a more positive perspective: The next galactic CCSN has already exploded. Using the predicted range of rates, one can, using a simple toy model of the galaxy assuming uniform star formation in the disk, estimate that $\sim 1000(\mathrm{SN}$ rate $) /\left(3.33 \times 10^{-2} \mathrm{SNe} \mathrm{yr}^{-1}\right) \mathrm{CCSNe}$ have already exploded and their photons, neutrinos, and gravitational waves (GWs) are on their way to Earth. The arrival of these messengers from the next galactic CCSN may be imminent. Due to intervening dust and gas, a significant fraction, perhaps $30-50 \%$, of these CCSNe will not be discovered in the optical, but will rather be seen as very luminous infrared sources and/or via their $\mathrm{X}$ - and $\gamma$-ray emission. Photons observed from CCSNe will initially carry information on the outer layers of the progenitor star, and only at late times allow us to probe central regions. Neutrinos and $\mathrm{GW}$, on the other hand, will provide plentiful information on the thermodynamics and dynamics of the supernova engine.

As shown by Lund et al. [28] (also seen in [65, 34, 15]), the SASI leads to characteristic time variations in the neutrino flux at $\sim 50-100 \mathrm{~Hz}$ that are observable by large Cherenkov detectors (see [66] and other contributions in this volume for a discussion of supernova neutrino detectors) for a galactic CCSN. If neutrino-driven

\footnotetext{
${ }^{2}$ We note that unlike the ratio of events at $20 \mathrm{~ms}$ to $100 \mathrm{~ms}$, the total number of events detected depends on the distance to the CCSN, which may not be determined (see $\$ 4$ ). However, given that the hierarchies are distinguishable for all models regardless of the total number of detected events (i.e. progenitor and EOS), this will not limit their distinguishability.
}

convection dominates, the preexplosion time variations of the neutrino flux may be expected to be characterized by smaller amplitude and higher frequency. Hence, the neutrino signal of the next galactic CCSN may observationally constrain the prominence of neutrino-driven convection and SASI.

GWs probe accelerated aspherical (of lowest-order quadrupole) mass-energy motions. Detailed reviews of the CCSN GW signature can be found in [25, 67]. In the case of a nonrotating or slowly rotating CCSN, GW emission will occur via $(i)$ prompt postbounce convection, which may grow on the negative entropy gradient left behind by the stalling shock, (ii) convection inside the protoneutron star, (iii) convection/SASI between the protoneutron star and the stalled shock, (iv) asymmetric neutrino emission, and $(v)$ aspherical outflows. The upcoming second-generation of $\mathrm{km}$-scale GW interferometers (Advanced LIGO [68], GEO-HF [69], Advanced Virgo [70], and KAGRA [71]) and third-generation instruments such as the Einstein Telescope [72] will be most sensitive to GWs in the frequency band $\sim 30-1000 \mathrm{~Hz}$, and will most likely detect GW emission from galactic and near extragalactic CC$\mathrm{SNe}$ (within, perhaps, $\sim 100 \mathrm{kpc}$ ). Emission processes (i-iii) lead to broadband emission in $\sim 50-1000 \mathrm{~Hz}$, while (iv) and ( $v$ ) are characterized by low-frequency $(\lesssim 10 \mathrm{~Hz}) \mathrm{GWs}$ and are thus less relevant for Earthbased detectors.

Studies investigating GW emission from convection/SASI (e.g., [27, 26, 73, 29, 23, 30]) have shown that the strongest component of GW emission comes from the deceleration of undershooting downflow plumes at the edge of the protoneutron star. As the region of deceleration is changing in the early postbounce phase, the time-frequency evolution of the GW signal observed from the next galactic CCSN will provide information on the early evolution of the structure, composition and thermodynamics of the lower postshock region, set, primarily, by the nuclear EOS and the postbounce accretion rate [27, 30]. This information encoded in GWs could be combined with information from neutrinos, which also probe the structure of the outer protoneutron star [58].

In the case of a rapidly spinning CCSN progenitor that may explode via the magnetorotational mechanism, core collapse proceeds approximately axisymmetrically [74, 75]. At bounce, the extreme acceleration experienced by the rotationally-deformed $(\ell=2, m=0)$ inner core leads to a strong burst of GWs, followed by quasi-periodic ring-down oscillations which continue for $\sim 10-15 \mathrm{~ms}$. At later postbounce times, rotational nonaxisymmetric $(m \neq 0)$ dynamics may develop due 
to a shear instability and lead to longer-term quasiperiodic GW emission at typically twice the rotation frequency of the protoneutron star [74, 75]. The characteristics of the GW signal due to dynamically-relevant rotation are rather distinct from those of the GW signal due to convection/SASI. If it is true that rapid rotation leads to magnetorotational explosions, while slowly rotating/nonrotating progenitor stars explode via the neutrino mechanism, the GW signal alone may be sufficient to determine the explosion mechanism via Bayesian model selection [76].

The neutrino signal seen from a rapidly rotating CCSN will depend on the inclination of the spin axis with respect to the observer (e.g., [34] and references therein): The rotational flattening of the protoneutron star leads to higher neutrino fluxes with harder spectra along the poles (i.e. the rotation axis) and to lower fluxes with softer spectra in equatorial regions. The hardness of the neutrino spectrum is also sensitive to the nuclear EOS (e.g., [58, 26]) and the inclination of the CCSN will not be known, unless it goes along with a gammaray burst, indicating a rotation axes aligned with the line of sight. Additional information carried in the GW signal can be used to break the degeneracy between rotation and EOS and constrain the unknown inclination angle. For example, if rotation is rapid, the protoneutron star ring-down oscillations seen in the GW signal go along with time variations in the $\bar{v}_{e}$ and $v_{x}$ fluxes at the same frequency that may be detected out to $O(1 \mathrm{kpc})$ distances [35]. If the CCSN spin axis is pointed at Earth, the $(\ell=2, m=0)$ GWs emitted by the ring-down oscillations will be suppressed, but the time-variations in the neutrino fluxes will be seen, as will be the subsequent GW emission by nonaxisymmetric dynamics, which is of $(\ell=2, m=2)$ character and peaks along the rotation axis [75]. Combining these different emissions will allow observers to constrain the inclination of the magnitude and orientation of the CCSN spin, in addition to helping to break the degeneracy between rotation and nuclear EOS.

The potential of combined analysis of GW and neutrino signals from the next galactic CCSN is tremendous. Much theoretical and modeling work is still needed to flesh out the details and parameter dependence (progenitor structure, rotation, EOS etc.) of both, $\mathrm{GW}$ and neutrino signals. This will require further improvements in modeling technology and efficiency to enable parameter studies in 3D. Progress is also needed in the development of parameter estimation approaches that will allow us to make statistical statements based on the combination of information carried in GWs and neutrinos.

\section{Acknowledgments}

We acknowledge helpful interactions at Neutrino 2012 with John Beacom, Ryan Patterson, Kate Scholberg, and Mark Vagins. This research is partially supported by NSF grant nos. AST-1212170, PHY1151197, PHY-1068881, and OCI-0905046, by the Alfred P. Sloan Foundation, and by the Sherman Fairchild Foundation. Some of the results presented here were obtained on supercomputers of the NSF XSEDE network under computer time allocation TG-PHY100033.

\section{References}

[1] K. Hirata, T. Kajita, M. Koshiba, M. Nakahata, Y. Oyama, Phys. Rev. Lett. 58 (1987) 1490.

[2] R. M. Bionta, G. Blewitt, C. B. Bratton, D. Casper, A. Ciocio, Phys. Rev. Lett.58 (1987) 1494.

[3] H. A. Bethe, Rev. Mod. Phys. 62 (1990) 801.

[4] S. A. Colgate, R. H. White, Astrophys. J.143 (1966) 626.

[5] W. D. Arnett, Canadian Journal of Physics 44 (1966) 2553.

[6] L. N. Ivanova, V. S. Imshennik, D. K. Nadezhin, NInfo 13 (1969) 3.

[7] J. R. Wilson, Astrophys. J.163 (1971) 209.

[8] H.-T. Janka, F. Hanke, L. Hüdepohl, A. Marek, B. Müller, M. Obergaulinger, Submitted to Prog. Th. Exp. Phys.; arXiv: 1211.1378

[9] A. Burrows, Rev. Mod. Phys. Collq. in press, arXiv:1210.4921

[10] K. Kotake, K. Sumiyoshi, S. Yamada, T. Takiwaki, T. Kuroda, Y. Suwa, H. Nagakura, Prog. Theo. Exp. Phys. 2012 (2012) 301.

[11] S. A. Colgate, M. H. Johnson, Phys. Rev. Lett.5 (1960) 235.

[12] H. A. Bethe, J. R. Wilson, Astrophys. J.295 (1985) 14.

[13] F. S. Kitaura, H.-T. Janka, W. Hillebrandt, Astron. Astrophys. 450 (2006) 345.

[14] J. W. Murphy, A. Burrows, Astrophys. J.688 (2008) 1159.

[15] A. Marek, H.-T. Janka, Astrophys. J.694 (2009) 664.

[16] B. Müller, H.-T. Janka, A. Marek, Astrophys. J.756 (2012) 84.

[17] B. Müller, H.-T. Janka, A. Heger, Submitted to the Astrophys. J., arXiv: 1205.7078

[18] J. Nordhaus, A. Burrows, A. Almgren, J. Bell, Astrophys. J.720 (2010) 694.

[19] F. Hanke, A. Marek, B. Müller, H.-T. Janka, Astrophys. J.755 (2012) 138.

[20] J. W. Murphy, J. C. Dolence, A. Burrows, Submitted to the Astrophys. J., arXiv:1205.3491

[21] J. C. Dolence, A. Burrows, J. W. Murphy, J. Nordhaus, Submitted to the Astrophys. J., arXiv:1210.5241

[22] A. Burrows, J. C. Dolence, J. W. Murphy, Astrophys. J.759 (2012) 5 .

[23] C. D. Ott, E. Abdikamalov, P. Moesta, R. Haas, S. Drasco, E. O'Connor, C. Reisswig, C. Meakin, E. Schnetter, Submitted to the Astrophys. J., arXiv:1210.6674

[24] S. W. Bruenn, A. Mezzacappa, W. R. Hix, E. J. Lentz, O. E. Bronson Messer, E. J. Lingerfelt, J. M. Blondin, E. Endeve, P. Marronetti, K. N. Yakunin, Submitted to Astrophys. J. Lett., arXiv: 1212.1747

[25] C. D. Ott, Class. Quantum Grav. 26 (2009) 063001.

[26] A. Marek, H.-T. Janka, E. Müller, Astron. Astrophys. 496 (2009) 475.

[27] J. W. Murphy, C. D. Ott, A. Burrows, Astrophys. J.707 (2009) 1173. 
[28] T. Lund, A. Wongwathanarat, H.-T. Janka, E. Müller, G. Raffelt, Phys. Rev. D.86 (2012) 105031

[29] E. Müller, H.-T. Janka, A. Wongwathanarat, Astron. Astrophys. 537 (2012) A63.

[30] B. Müller, H.-T. Janka, A. Marek, Submitted to the Astrophys. J., arXiv:1210.6984

[31] M. Modjaz, Astron. Nachr. 332 (2011) 434.

[32] G. S. Bisnovatyi-Kogan, Astron. Zh. 47 (1970) 813.

[33] A. Burrows, L. Dessart, E. Livne, C. D. Ott, J. Murphy, Astrophys. J.664 (2007) 416.

[34] C. D. Ott, A. Burrows, L. Dessart, E. Livne, Astrophys. J.685 (2008) 1069.

[35] C. D. Ott, E. Abdikamalov, E. O’Connor, C. Reisswig, R. Haas, P. Kalmus, S. Drasco, A. Burrows, E. Schnetter, Phys. Rev. D.86 (2012) 024026.

[36] H. Duan, G. M. Fuller, Y.-Z. Qian, Ann. Rev. Nuc. Part. Sc. 60 (2010) 569.

[37] S. Mikheev, A. Smirnov, Sov.J.Nucl.Phys. 42 (1985) 913.

[38] Y. Suwa, K. Kotake, T. Takiwaki, M. Liebendörfer, K. Sato, Astrophys. J.738 (2011) 165

[39] S. Chakraborty, T. Fischer, A. Mirizzi, N. Saviano, R. Tomàs, Phys. Rev. Lett. 107 (2011) 151101

[40] S. Sarikas, G. G. Raffelt, L. Hüdepohl, H.-T. Janka, Phys. Rev. Lett.108 (2012) 061101.

[41] B. Dasgupta, E. P. O’Connor, C. D. Ott, Phys. Rev. D.85 (2012) 065008.

[42] J. F. Cherry, J. Carlson, A. Friedland, G. M. Fuller, A. Vlasenko, Phys. Rev. Lett.108 (2012) 261104.

[43] M. Herant, W. Benz, W. R. Hix, C. L. Fryer, S. A. Colgate, Astrophys. J.435 (1994) 339.

[44] A. Burrows, J. Hayes, B. A. Fryxell, Astrophys. J.450 (1995) 830.

[45] H.-T. Janka, E. Müller, Astron. Astrophys. 306 (1996) 167.

[46] J. M. Blondin, A. Mezzacappa, C. DeMarino, Astrophys. J.584 (2003) 971.

[47] L. Scheck, H.-T. Janka, T. Foglizzo, K. Kifonidis, Astron. Astrophys. 477 (2008) 931.

[48] T. Foglizzo, L. Scheck, H.-T. Janka, Astrophys. J.652 (2006) 1436.

[49] W. Iwakami, K. Kotake, N. Ohnishi, S. Yamada, K. Sawada, Astrophys. J.700 (2009) 232.

[50] W. Iwakami, K. Kotake, N. Ohnishi, S. Yamada, K. Sawada, Astrophys. J.678 (2008) 1207.

[51] W. D. Arnett, C. Meakin, Astrophys. J.733 (2011) 78.

[52] J. Guilet, J. Sato, T. Foglizzo, Astrophys. J.713 (2010) 1350.

[53] S. Couch, submitted to the Astrophys. J., arXiv:1212.0010

[54] T. Kuroda, K. Kotake, T. Takiwaki, Astrophys. J.755 (2012) 11.

[55] T. Takiwaki, K. Kotake, Y. Suwa, Astrophys. J.749 (2012) 98.

[56] P. D. Serpico, S. Chakraborty, T. Fischer, L. Hüdepohl, H.-T. Janka, A. Mirizzi, Phys. Rev. D.85 (2012) 085031.

[57] A. S. Dighe, A. Y. Smirnov, Phys. Rev. D.62 (2000) 033007.

[58] E. O'Connor, C. D. Ott, Astrophys. J. in press, arXiv:1207.1100

[59] R. Abbasi et al. [IceCube Collaboration], Astron. Astrophys. 535 (2011) A109.

[60] S. E. Woosley, A. Heger, Phys. Rep. 442 (2007) 269

[61] J. M. Lattimer, F. D. Swesty, Nucl. Phys. A 535 (1991) 331.

[62] H. Shen, H. Toki, K. Oyamatsu, K. Sumiyoshi, Astrophys. J. Supp. Ser. 197 (2011) 20.

[63] G. A. Tammann, W. Loeffler, A. Schroeder, Astrophys. J. Supp. Ser. 92 (1994) 487.

[64] E. Cappellaro, R. Evans, M. Turatto, Astron. Astrophys. 351 (1999) 459.

[65] T. D. Brandt, A. Burrows, C. D. Ott, E. Livne, Astrophys. J.728 (2011) 8 .

[66] K. Scholberg, Ann. Rev. Nuc. Part. Sc. 62 (2012) 81.
[67] K. Kotake, submitted to a special issue of Comptes Rendus Physique "Gravitational Waves (from detectors to astrophysics)", arXiv:1110.5107

[68] G. M. Harry, LIGO Scientific Collaboration, Class. Quantum Grav. 27 (2010) 084006.

[69] H. Grote, the LIGO Scientific Collaboration, Class. Quantum Grav. 27 (2010) 084003.

[70] T. Accadia et al. (Virgo Collaboration), Class. Quantum Grav. 28 (2011) 114002

[71] K. Somiya (for the KAGRA collaboration), Class. Quantum Grav. 29 (2012) 124007.

[72] M. Punturo, et al., Class. Quantum Grav. 27 (2010) 194002.

[73] K. N. Yakunin, P. Marronetti, A. Mezzacappa, S. W. Bruenn, C.T. Lee, M. A. Chertkow, W. R. Hix, J. M. Blondin, E. J. Lentz, O. E. Bronson Messer, S. Yoshida, Class. Quantum Grav. 27 (2010) 194005.

[74] C. D. Ott, H. Dimmelmeier, A. Marek, H.-T. Janka, I. Hawke, B. Zink, E. Schnetter, Phys. Rev. Lett.98 (2007) 261101.

[75] S. Scheidegger, R. Käppeli, S. C. Whitehouse, T. Fischer, M. Liebendörfer, Astron. Astrophys. 514 (2010) A51.

[76] J. Logue, C. D. Ott, I. S. Heng, P. Kalmus, J. Scargill, Phys. Rev. D.86 (2012) 044023 . 